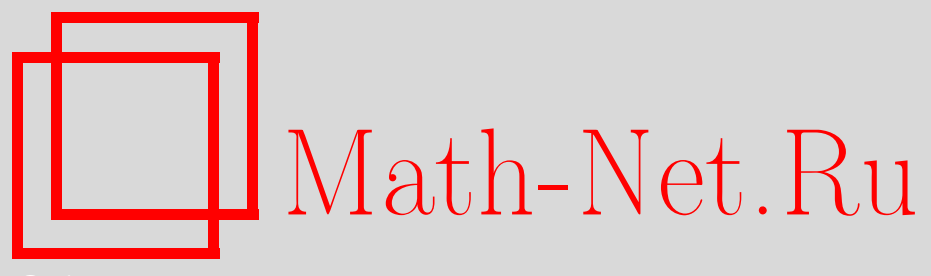

И. В. Белошапка, О представлениях группы Гейзенберга матриц с одним целым и двумя вещественными коэффициентами, УМН, 2014, том 69, выпуск 5, 161-162

DOI: https://doi.org/10.4213/rm9623

Использование Общероссийского математического портала Math-Net.Ru подразумевает, что вы прочитали и согласны с пользовательским соглашением http://www . mathnet.ru/rus/agreement

Параметры загрузки:

IP : 54.92 .164 .108

26 апреля 2023 г., 13:17:02

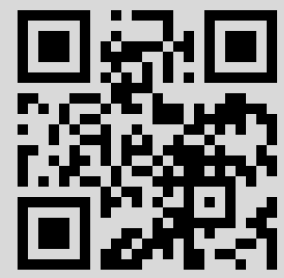




\section{О представлениях группы Гейзенберга матриц с одним целым и двумя вещественными коэффициентами}

\section{И. В. Белошапка}

Пусть $H, P, C$ - абелевы группы, $\langle\cdot, \cdot\rangle$ - билинейное отображение из $H \times P$ в $C$. Группой Гейзенберга $G$ мы называем множество $H \times P \times C$ с законом композиции $\left(h_{1}, p_{1}, c_{1}\right) \cdot\left(h_{2}, p_{2}, c_{2}\right)=\left(h_{1}+h_{2}, p_{1}+p_{2}, c_{1}+c_{2}+h_{1} p_{2}\right)$.

Пусть $G_{\chi}=H_{\chi} P C$, где $H_{\chi}=\left\{h \in H: \chi_{C}(\langle h, p\rangle)=1\right.$ для всех $\left.p \in P\right\}$ и $\chi_{C}-$ характер подгруппы $C$. Тогда $G_{\chi}$ - нормальный делитель группы $G$, он зависит только от $\chi_{C}$. Пусть далее $H \simeq \mathbb{Z}, P \simeq C \simeq \mathbb{R}$ и $\chi_{P}(p)=e^{2 \pi i s p}, \chi_{C}(c)=e^{2 \pi i t c}-$ характеры подгрупп $P, C$ соответственно, $s, t \in \mathbb{C}$. Тогда подгруппа $H_{\chi}$ тривиальна, если $t \neq 0$, и равна $\mathbb{Z}$, если $t=0$.

ОПредЕлЕниЕ 1 . Пусть $V_{\chi}$ - пространство комплекснозначных функций $f$ на $G$ со свойствами: (i) $f(h g)=\chi(h) f(g), h \in G_{\chi}$; (ii) носитель функции $f$ содержится в конечном числе классов смежности группы $G: \operatorname{Supp}(f) \subset \bigcup_{i \in I} h_{i} G,|I|<\infty$.

Правые сдвиги определяют представление $\pi_{\chi}$ в пространстве $V_{\chi}=\operatorname{ind}_{G_{\chi}}^{G}(\chi)$.

Теорема 1. І. Если $t \neq 0$, то: 1) $V_{\chi}$ не содержат нетривиалъных относительно действия группы $G$ подпространств; 2) $V_{\chi}$ бесконечномерны; 3) линейные преобразования, коммутирующие с действием группь $G$, скалярны; 4) если $\chi_{1} \sim\left(t_{1}, s_{1}\right)$, $\chi_{2} \sim\left(t_{2}, s_{2}\right)$, по $\pi_{\chi_{1}} \sim \pi_{\chi_{2}}$ тогда и только тогда, когда существует $h \in \mathbb{Z}$ такое, что $t_{1}=t_{2}, s_{1}=s_{2}+h t_{1}$.

II. Eсли $t=0$, mo $G_{\chi}=G u V_{\chi}$-одномерное представление группъ $G$.

Теорема доказывается незначительными изменениями рассуждений в доказательстве аналогичного утверждения в [1].

ОПределение 2 [2]. Пусть $\rho: G \rightarrow \operatorname{End}(V)$ - представление группы $G$, где $V-$ счетномерное комплексное пространство, тогда $V(K, \chi)$ - весовое подпространство представления $V$, если $V(K, \chi)=\{v \in V \mid \rho(k) v=\chi(k) v \forall k \in K\}$, где $K \subset G$ подгруппа $G$ и $\chi: K \rightarrow \mathbb{C}^{*}$. Представление $\rho$ группы $G$ обладает конечным весом, если существуют подгруппа $K \subset G$ и характер $\chi: K \rightarrow \mathbb{C}^{*}$ этой подгруппы такие, что: 1) $V(K, \chi) \neq(0) ; 2) \operatorname{dim}_{\mathbb{C}} V(K, \chi)<\infty$.

Следуя [2], формулируем следующую теорему.

Теорема 2. Пусть $\rho$ - представление группы $G$ в счетномерном комплексном пространстве $V$ с конечным весом относительно подгруппы $P C$, тогда существует характер $\chi: P C \rightarrow \mathbb{C}^{*}$ такой, что $\pi \sim \pi_{\chi}$, m.е. представление $\rho$ мономиально.

Определим след $\operatorname{Tr} \pi_{\chi}(g)$ представления $\pi_{\chi}$ как комплекснозначную функцию на группе, вычисляемую как след оператора представления $\pi_{\chi}(g)$ в базисе $\delta_{m}, m \in \mathbb{Z}$, пространства $D(\mathbb{Z})$. Легко видеть, что так определенный ряд никогда не сходится. Расширим группу $G: \widehat{G}=G \rtimes A$ как полупрямое представление, где $A=$ $\operatorname{Hom}(H, P) \simeq \mathbb{R}$. Тогда $A$ действует на $G$ по формуле: $\forall a \in A \quad a(h, p, c):=(h, p+$ $a h, c+a h(h-1) / 2), h \in H, p \in P, c \in C$. Представление $\pi_{\chi}$ группы $G$ можно продолжить как представление $\widehat{\pi}_{\chi}$ группы $\widehat{G}$ на том же пространстве: $\widehat{\pi}_{\chi}:=\pi_{\chi} \otimes \chi_{A}$ (см. [1]), где $\chi_{A}$ - характер подгруппы $A, \chi_{A}(a)=e^{2 \pi i u a}, \widehat{\chi}=\left(\chi, \chi_{A}\right)-$ характер группы $\widehat{G}$. Действие группы $\widehat{G}$ будет таким:

$\widehat{\pi}_{\chi}(m, p, c, a) \delta_{n}=\exp \left\{2 \pi i\left(t c+s p+s a(n-m)+\frac{1}{2} t a(n-m)(n-m-1)+t(n-m) p\right)\right\} \delta_{n-m}$.

Работа выполнена при поддержке РФФИ (грант № 14-01-00160_а).

DOI: $10.4213 / \mathrm{rm} 9623$ 
Тогда след представления $\widehat{\pi}_{\chi}(g)$ группы $\widehat{G}$ определен при $\operatorname{Re}(a t)<0$ и равен нулю при $m \neq 0, m \in H$. Из рассуждений о спектрах подгрупп получаем, что $\widehat{\pi}_{\widehat{\chi}_{1}} \sim \widehat{\pi}_{\widehat{\chi}_{2}}$ тогда и только тогда, когда существует $h \in H$ такое, что

$$
t_{1}=t_{2}, \quad s_{1}=s_{2}+h t_{1}, \quad u_{1}=u_{2}+h s_{1}+\frac{1}{2} h(h-1) t_{1} .
$$

Обозначим $\mathbb{M}_{G}$ пространство модулей неприводимых индуцированных представлений: $(t, s, u) \in \mathbb{C}^{*} \times \mathbb{C} \times \mathbb{C} / \sim$, где $\widehat{\pi}_{\hat{\chi}_{1}} \sim \widehat{\pi}_{\hat{\chi}_{2}}$, если найдется $h \in \mathbb{Z}$ такое, что выполнено (1).

ПредлОЖЕНИЕ 1. $\widehat{\pi}_{\widehat{\chi}_{1}} \sim \widehat{\pi}_{\widehat{\chi}_{2}} \Leftrightarrow \operatorname{Tr} \widehat{\pi}_{\widehat{\chi}_{1}}(g)=\operatorname{Tr} \widehat{\pi}_{\widehat{\chi}_{2}}(g) \forall g \in \widehat{G} n p u \operatorname{Re}\left(a t_{1}\right), \operatorname{Re}\left(a t_{2}\right)<0$.

ДокАЗАТЕльство. $\Rightarrow$ Проверяется непосредственно.

$\Leftarrow$ Рассмотрим отображение $F:(t, s, u) \mapsto\left(e^{2 \pi i t}, e^{2 \pi i s}, e^{2 \pi i u}, e^{2 \pi^{2} i t}, e^{2 \pi^{2} i s}, e^{2 \pi^{2} i u}\right)=$ $\left(\lambda, z, \tau, \lambda^{\prime}, z^{\prime}, \tau^{\prime}\right)$. Считаем, что $t, s, \pi t, \pi s$ иррациональны, $\operatorname{Im} t \neq 0$, иначе добиваемся этого заменой $F\left(c_{1} t, c_{2} s, u\right)=\widehat{F}(t, s, u)$. Тогда

$$
\begin{aligned}
\operatorname{Tr} \widehat{\pi}_{\hat{\chi}}(0, p, c, a) & =e^{2 \pi i(t c+s p+a u)} \sum_{m \in \mathbb{Z}} e^{2 \pi i(t m p+\operatorname{sam}+\operatorname{tam}(m-1) / 2)} \\
& =\tau^{a} \lambda^{c} z^{p} \sum_{m \in \mathbb{Z}} z^{a m} \lambda^{m p+a m(m-1) / 2} .
\end{aligned}
$$

Рассуждения в [1] показывают, что комплексные числа $(\lambda, z, \tau)$ определяют линейное расслоение над семейством эллиптических кривых $E_{\lambda}$, где $\lambda \in \mathbb{C}^{*}(|\lambda| \neq 1$, так как $\operatorname{Im} t \neq 0), z$ лежит на эллиптической кривой $E_{\lambda} \simeq \mathbb{C}^{*} / \lambda^{\mathbb{Z}}, z \mapsto z \lambda^{h}, h \in \mathbb{Z}$. Коцикл $\tau \mapsto \tau z^{h} \lambda^{h(h-1) / 2}$ определяет расслоения $L$ степени 1 и $L^{\otimes k}$ степени $k, k \in \mathbb{Z}$, на эллиптической кривой $E_{\lambda}$. Функции $\theta_{p, k}(\lambda, z)=z^{p} \sum_{m \in \mathbb{Z}} z^{k m} \lambda^{m p+k m(m-1) / 2}$ при $0 \leqslant p \leqslant k-1$ составляют базис голоморфных сечений расслоения $L^{\otimes k}$ на $E_{\lambda}$. Имеем:

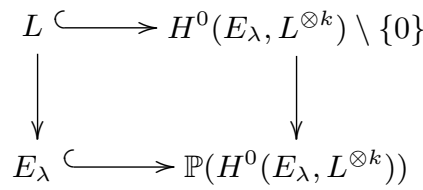

Это дает вложение пространства модулей $\mathbb{M}_{G}$ в аффинное пространство и равенства $\lambda_{1}=\lambda_{2}, z_{1}=z_{2} \lambda^{h}, \tau_{1}=\tau_{2} z_{1}^{h} \lambda^{h(h-1) / 2}$ и $\lambda_{1}^{\prime}=\lambda_{2}^{\prime}, z_{1}^{\prime}=z_{2}^{\prime}\left(\lambda_{1}^{\prime}\right)^{h}, \tau_{1}^{\prime}=\tau_{2}^{\prime}\left(z_{1}^{\prime}\right)^{h}\left(\lambda_{1}^{\prime}\right)^{h(h-1) / 2}$, откуда получаем: $t_{1}=t_{2}, s_{1}=s_{2}+h t_{1}, u_{1}=u_{2}+h s_{1}+h(h-1) t_{1} / 2$, что и требовалось.

ПреДЛОЖЕНИЕ 2. 1) При $t \rightarrow 0$ представление $\pi_{\chi}$ раскладывается в бесконечную прямую сумму одномерных представлений $\mu=\mu^{\prime} \otimes \chi_{P} \otimes 1$, где $\mu^{\prime}$ пробегает все характеры подгруппы $H$.

2) Элементы $(h, p, c, a) u\left(h^{\prime}, p^{\prime}, c^{\prime}, a^{\prime}\right)$, где $h, h^{\prime} \neq 0$, сопряжены в $\widehat{G}$, если $h=h^{\prime}$, $a=a^{\prime}$. Eсли $h=0$, то $(0, p, c, a)$ сопрлжен $\left(0, p^{\prime}, c^{\prime}, a^{\prime}\right)$ тогда и толъко тогда, когда существует $n \in \mathbb{Z}$ такое, ито $p^{\prime}=p+$ an, $c^{\prime}=c+p n+a n(n-1) / 2, a=a^{\prime}$.

3) Если элементы $g=(0, p, c, a) u g^{\prime}=\left(0, p^{\prime}, c^{\prime}, a^{\prime}\right)$ не сопряжены в $\widehat{G}$, то

$$
\int_{\mathbb{M}_{G}} \operatorname{Tr} \widehat{\pi}_{\widehat{\chi}}(g) \overline{\operatorname{Tr} \widehat{\pi}_{\widehat{\chi}}\left(g^{\prime}\right)} d \mu=0 .
$$

4) $\chi_{P}(p) \chi_{C}(c)=\lim _{l \rightarrow \infty} \operatorname{Tr} \widehat{\pi}_{\chi}(0, p, c, l a)$ npu $\operatorname{Re}(a t)<0, p \in P, c \in C, l \in \mathbb{N}$.

\section{Список литературы}

[1] A. N. Parshin, Lectures on representations of discrete Heisenberg groups, Humboldt Univ., Berlin, 2010. [2] С. А. Арналь, А. Н. Паршин, Матем. заметки, 92:3 (2012), 323-330.

И. В. Белошапка (I. V. Beloshapka)

Московский государственный университет им. М. В. Ломоносова

E-mail: i-beloshapka@yandex.ru
Представлено С. Ю. Немировским Принято редколлегией 03.08.2014 